\title{
Methods of neutron source strength measurements
}

\section{Nikolai Moiseev}

D.I.Mendeleyev Institute for Metrology

19 Moskovsky pr., Saint-Petersburg,Russia

E-mail: n.n.moiseev@vniim.ru

\section{Igor Kharitonov}

D.I.Mendeleyev Institute for Metrology

19 Moskovsky pr., Saint-Petersburg,Russia

E-mail: i.a.kharitonov@vniim.ru

The methods used at the D.I.Mendeleyev Institute for Metrology for the absolute measurement of emission rates of radionuclide neutron sources are described. All methods are based on different physical principles: associated charged particles counting, manganese sulphate activation, gold foil activation and thermal flux intensity measurement. The standard uncertainties of the results do not exceed $1.2 \%$ for each of the methods. The result obtained using different methods are in good agreement.

International Workshop on Fast Neutron Detectors

University of Cape Town, South Africa

April 3-6, 2006 


\section{Introduction}

The emission rate, i.e. the number of neutrons emitted into the $4 \pi$ solid angle, is the basic quantity characterising radionuclide neutron sources. In this paper, the equipment and the techniques used at the D.I. Mendeleyev Institute for Metrology (VNIIM) for the measurement of the emission rates are described.

The VNIIM laboratory for neutron measurements operates facilities for accurate measuring neutron emission rates using three independent absolute methods $[1,2,3,4]$. The relative standard uncertainties $(k=1)$ do not exceed $0.7 \%$ for each of these methods.

\section{Equipment and experimental techniques}

\subsection{The manganese bath method}

This method is used in almost all national laboratories engaged in neutron measurements. It is therefore not described it here in detail. The VNIIM manganese bath is a cylindrical tank 0.85 $\mathrm{cm}$ in diameter and $0.95 \mathrm{~cm}$ in height, which is made of stainless steel and filled with $\mathrm{MnSO}_{4}$ solution. The bath has no system for circulation of the solution. The photons resulting from the decay of ${ }^{56} \mathrm{Mn}$ are detected with a scintillation detector which is placed in the bath after removing the source from the bath and stirring of the manganese sulphate solution.

The correction for leakage of neutrons from the moderator is determined experimentally. In contrast, the corrections for capture of thermal neutrons by impurities and for capture of thermal and fast neutrons by sulphur and oxygen nuclei and structural materials is calculated.

The equipment is used to measure the neutron emission rate of radionuclide sources in the range from $10^{5} \mathrm{~s}^{-1}$ to $10^{8} \mathrm{~s}^{-1}$ with a relative standard uncertainty $(k=1)$ of $0.55 \%$.

\subsection{The gold foil activation method}

This method is a well-known method too, although is not used as widely as the manganese bath. No national metrological laboratory employed this method in the key-comparison CCRI(III)-K.9.Am-Be (1999 - 2005) and only two laboratories, PTB and VNIIM, used it in the comparison CCRI(III)-K.9.Cf-252 (1978 - 1984) [5].

If a neutron source is placed in the centre of a relatively big moderator (in present case, water), its emission rate $B$ can be determined by

$$
B=4 \pi \int_{0}^{\infty} n_{\mathrm{H}} \sigma_{\mathrm{H}} \varphi_{\mathrm{T}}(r) r^{2} \mathrm{~d} r
$$

where $n_{\mathrm{H}}, \sigma_{\mathrm{H}}$ and $\varphi_{\mathrm{T}}(r)$ denote the number of hydrogen nuclei per unit volume, the cross section for capture of thermal neutrons by hydrogen and the thermal neutron fluence rate in the moderator at the distance $r$ from the source centre, respectively. Thus, the emission rate $B$ can be determined from the known cross-section $\sigma_{\mathrm{H}}$ and $\varphi_{\mathrm{T}}(r)$. 
The VNIIM installation is a cylinderical stainless steel tank, $110 \mathrm{~cm}$ in diameter and $130 \mathrm{~cm}$ in height, which is filled with bi-distilled water. Gold activation detectors are used for the measurement of the thermal neutron distribution $\varphi_{T}(r)$. One of the special design features of this equipment is the system for the displacement of the detector and the source support. This system causes a minimum distortion of the neutron field. The position of the gold activation detector relative to the source centre is determiend with an uncertainty less than $0.1 \mathrm{~mm}$.

The equipment is used to measure the neutron emission rates of radionuclide sources in the range from $10^{6} \mathrm{~s}^{-1}$ to $5 \cdot 10^{7} \mathrm{~s}^{-1}$ with a relative standard uncertainty $(k=1)$ of $0.55 \%$. The activity of ${ }^{198} \mathrm{Au}$ in the gold foils is measured using the $\beta-\gamma$ and $4 \pi \beta-\gamma$ coincidence facilities.

The other two methods are described here in more detail because they are not so widely known.

\subsection{Associated particle method}

If the ratio between the number of neutrons and charged particles emitted in the neutron producing reaction as well as the spatial distribution of reaction products is known, the neutron emission rate can be determined by a measurement of the number of associated particles emitted into a certain solid angle $\Omega$. The neutron fluence value can be calculated by the equation

$$
B=\frac{N}{\Omega \varepsilon k(\vartheta)} \eta
$$

where $N, \varepsilon, k(\vartheta), \Omega$ and $\eta$ denote the associated radiation counting rate, the efficiency of the associated radiation detector, the asymmetry coefficient of the associated radiation, the solid angle in which the associated radiation is recorded and the ratio between the number of neutrons and that of associated particles, respectively.

Unfortunately, this well-known technique can not be used directly for the determination of radionuclide neutron sources emission rate. However, the emission rate of radionuclide sources can be determined by comparison with the emission rate of a neutron generator using a 'comparator' device.

The VNIIM neutron laboratory has a unique equipment for this purpose - a spherical moderator $4 \mathrm{~m}$ in diameter (see Fig. 1). The sphere is made of pure reactor graphite and consists of twenty horizontal layers with about two thousand blocks. There is a spherical cavity $40 \mathrm{~cm}$ in diameter at the centre of the moderator. A vertical channel $90 \mathrm{~mm}$ in diameter is used to place the radionuclide neutron sources at the centre of the cavity. The $175 \mathrm{~mm}$ horizontal channel is used to place the neutron generator target at the centre of the cavity. Two other $90 \mathrm{~mm}$ diameter channels transverse the sphere close to the central cavity with an inclination of $24^{\circ}$ to the horizontal. All open space in these channels is filled with graphite inserts. A thermal neutron detector is mounted in one of the inserts of each channel. This detector can be displaced in the distance range from $27.5 \mathrm{~cm}$ to $202 \mathrm{~cm}$ from the centre of the moderator sphere. 


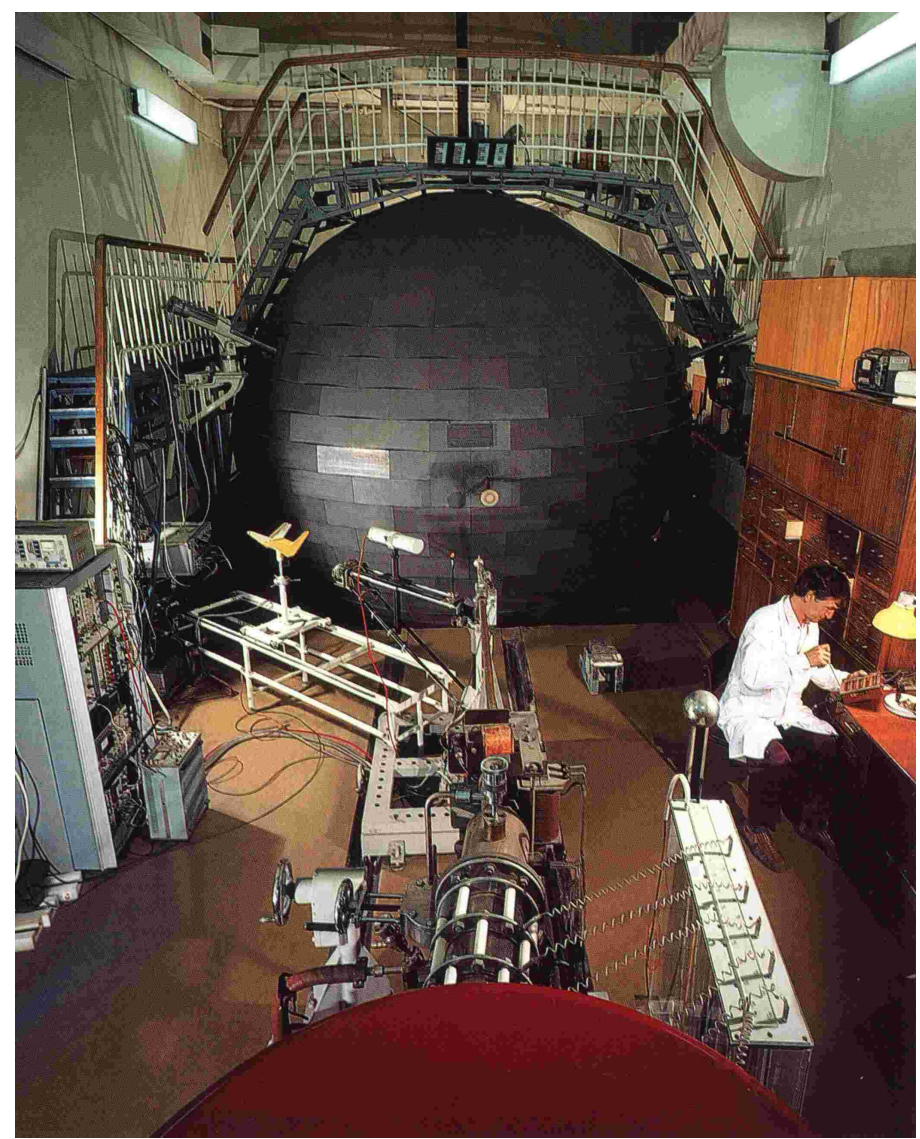

Fig 1: Experimental set-up with the huge graphite moderator sphere in the rear, the fast neutron generator in front and one of the authors (NM) in the middle

The source located in the moderator centre emits fast neutrons which interact with carbon nuclei and are moderated to the thermal energies. Since the diffusion length of thermal neutrons in graphite is about $52 \mathrm{~cm}$ and the moderator radius is about $2 \mathrm{~m}$, this moderator can be considered quasi-infinite. The spatial distribution of thermal neutrons in the moderator volume can be studied by displacing the thermal neutron detector. The detector is designed for a constant efficiency in the fluence rate range from $0.1 \mathrm{~s}^{-1} \mathrm{~cm}^{-2}$ to $10^{5} \mathrm{~s}^{-1} \mathrm{~cm}^{-2}$.

At VNIIM, monoenergetic neutron reference fields with energies of $2.5 \mathrm{MeV}$ and $14 \mathrm{MeV}$ are produced with a special neutron generator. This generator can also be used with the moderator sphere. The target chamber provides the possibility to determine the emission rate of monoenergetic neutrons by counting the associated charged particles. In accordance with its metrological purposes, the neutron generator has a number of special features:

The target chamber construction ensures a minimum distortion of the neutron field and makes it possible to count the associated particles at a fixed solid angle. The chamber is a stainless steel sphere, $50 \mathrm{~mm}$ in diameter and $0.8 \mathrm{~mm}$ in thickness. The neutron production target, the semiconductor detector for counting the associated charged particles and the apertures defining the solid angle are mounted in a special tube in the chamber. The distance between the target and the aperture is fixed and can be measured with very small 
uncertainty. Thus, the reproducibility of the geometric factor is ensured even when the target or detector are replaced.

The analysing magnet provides separation of the different charge states of deuterium ions which is required to produce monoenergetic neutron radiation.

The neutron generator is operated without target cooling. This limits the ion current ( $20 \mu \mathrm{A}$ at maximum) and consequently the neutron emission rate but it allows an essential decrease in distortion and attenuation of the initial neutron radiation.

The generator can be moved on rails and is equipped with a beam tube $2.5 \mathrm{~m}$ in length. This makes it possible to place the neutron generator target chamber in the central cavity of moderator (see Fig. 2).

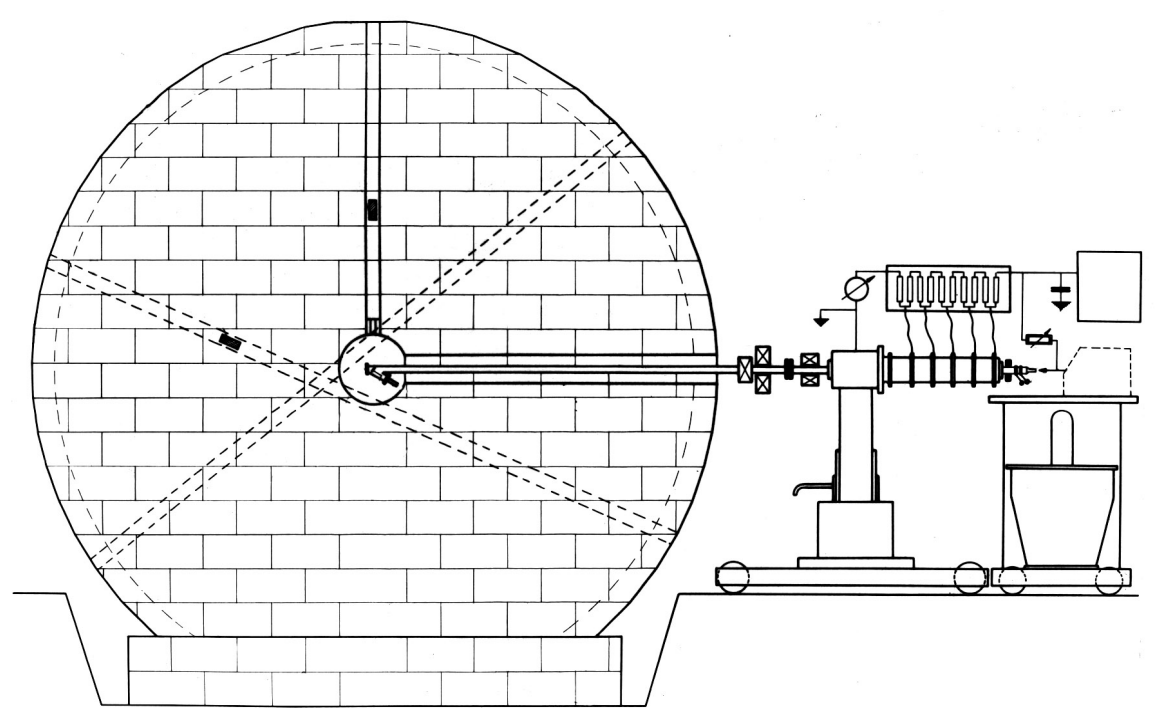

Fig 2: Cross section of the spherical graphite moderator, $4 \mathrm{~m}$ in diameter, with a horizontal channel for inserting the target of the neutron generator in the centre, a vertical channel for inserting sealed sources from the top and two channels for measuring the fluence of thermal neutrons as a function of the distance to the centre.

These features facilitates the comparison of the neutron emission rate of radionuclide neutron sources with the neutron fluence measured with the associated particle technique:

The ratio between the moderation length and thermal neutron diffusion length in graphite is such that neutron leakage does not depend on the neutrons energy in the range up to $20 \mathrm{MeV}$

There is a spherical layer in the moderator at the distance of about $79 \mathrm{~cm}$ from the centre where the ratio of thermal neutron fluence to neutron source strength does not depend on the initial neutron energy in the range from 0.5 to $20 \mathrm{MeV}$ (see Fig. 3).

There are no reactions in graphite increasing the number of neutrons up to energies of about $20 \mathrm{MeV}$;

The facility is practically insensitive to the angular asymmetry of the primary neutron field. 

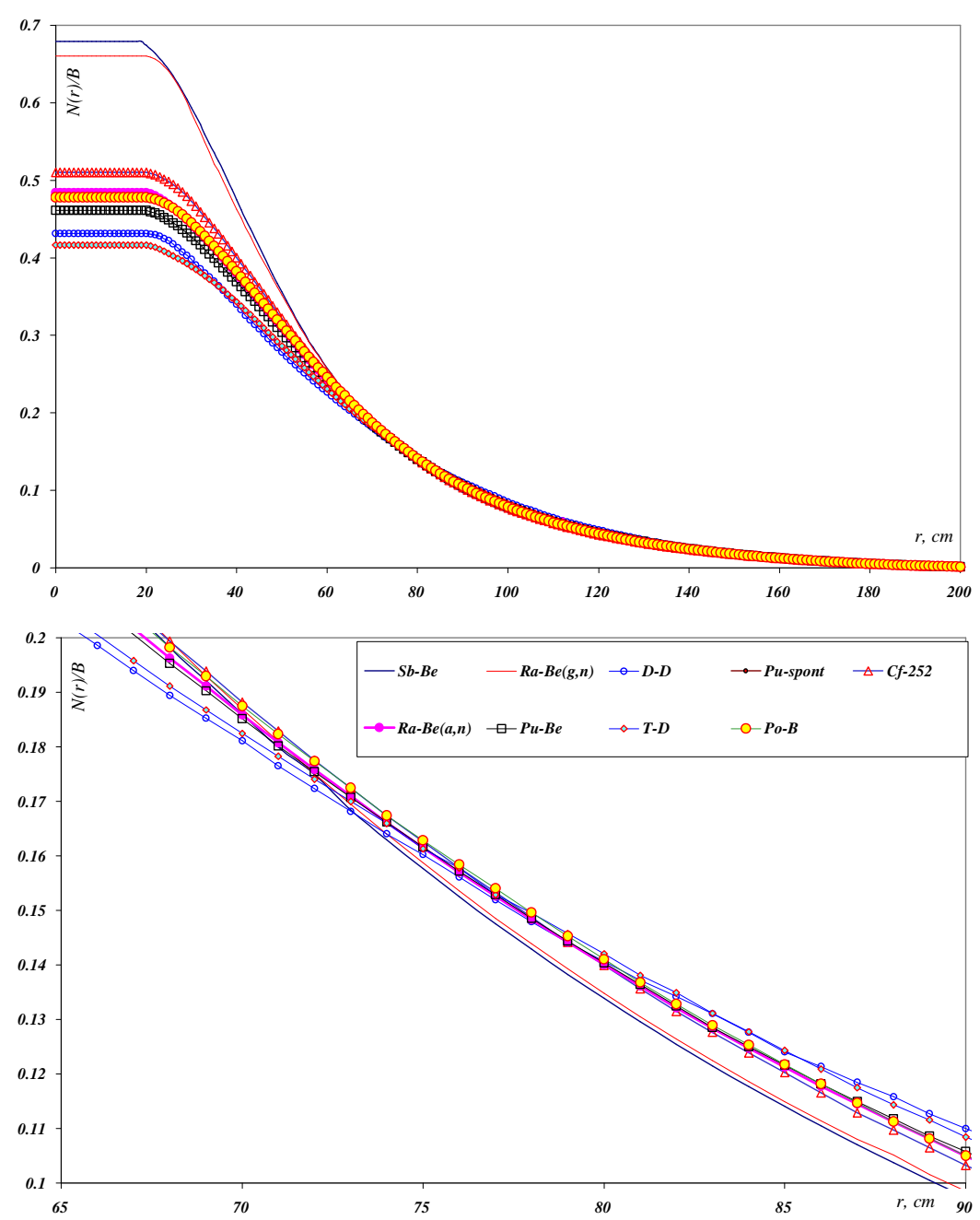

Fig 3: The count rate of the thermal neutron detector $N(r)$ as a function of the distance $r$ to the centre as measured for selected neutron sources, normalised to unit neutron emission rate $B$. The expanded diagram clearly shows an intersection of all curves except that for the $\mathrm{Sb}-\mathrm{Be}$ and $\operatorname{Ra}-\mathrm{Be}(\gamma, \mathrm{n})$ sources at about $78 \mathrm{~cm}$, independent of the spectral neutron fluence of the source.

The total number of neutrons in the sphere is proportional to the integral $S$ of the count rate $N(r)$ of the thermal neutron detector over the sphere volume.

$$
S=\int_{a_{1}}^{a_{2}} N(r) r^{2} \mathrm{~d} r
$$

Here $a_{1}=0.2 \mathrm{~m}$ and $a_{2}=2 \mathrm{~m}$ denote the of the radius of the central cavity with the neutron source at its centre and the radius of the moderator sphere, respectively. The source strength $B$ of a radionuclide neutron source can be calculated from the source strength $B_{0}$ of the T(d,n ${ }^{4} \mathrm{He}$ neutron source measured using the associated particle technique and the integrals $S_{0}$ and $S$ of the 
counting rates of the thermal neutron detector measured for the $\mathrm{T}(\mathrm{d}, \mathrm{n})$ source and the radionuclide neutron source.

$$
B=B_{0} \frac{S}{S_{0}} \frac{k_{(\mathrm{n}, \alpha)}}{k_{0,(\mathrm{n}, \alpha)}}
$$

The contribution of epithermal neutrons to the integrals $S$ and $S_{0}$ is determined by the Cd difference method.

The correction $k_{(\mathrm{n}, \alpha)}$ for loss of neutrons in the graphite due to $(\mathrm{n}, \alpha)$-reactions were calculated using the Monte-Carlo method and also determined experimentally for several types of sources using a method based on incomplete moderation.

For these measurements, a radionuclide neutron source in a spherical polyethylene moderator with radius $r$ is placed in the cavity of the graphite sphere. The counting rate $N(r)$ of a thermal neutron detector located at the point of 'constant sensitivity' is measured. With the increase of the polyethylene moderator radius $r$, the number of neutrons emitted from its surface with an energy exceeding the threshold of the reaction ${ }^{12} \mathrm{C}(\mathrm{n}, \alpha)-{ }^{9} \mathrm{Be}$ is reduced. Extrapolating the dependence of the quantity $\left(1-N(r) / N_{0}\right)$ to zero thickness of the moderator, the emission rate of a 'negative source' in relative units is obtained, i.e. the value of the correction for absorption of neutrons by graphite. Here $N_{0}$ denotes the counting rate measured for the source without the moderator.

For monoenergetic neutrons with an energy of $14.1 \mathrm{MeV}$, the correction for absorption in graphite was determined in a separate experiment. Since the threshold of the reaction ${ }^{12} \mathrm{C}(\mathrm{n}, \alpha){ }^{9} \mathrm{Be}$ is at $6.18 \mathrm{MeV}$, this effect does not contribute for neutrons from the reaction $\mathrm{D}(\mathrm{d}, \mathrm{n})^{3} \mathrm{He}$. Therefore the correction for $14.1 \mathrm{MeV}$ neutrons can be obtained from the ratio of counting rates $N_{\mathrm{T}(\mathrm{d}, \mathrm{n})}$ and $N_{\mathrm{D}(\mathrm{d}, \mathrm{n})}$ measured in the graphite sphere for the reactions $\mathrm{T}(\mathrm{d}, \mathrm{n})^{4} \mathrm{He}$ and $\mathrm{D}(\mathrm{d}, \mathrm{n})^{3} \mathrm{He}$ and from the ratio of emission rates $B_{\mathrm{T}(\mathrm{d}, \mathrm{n})}$ and $B_{\mathrm{T}(\mathrm{d}, \mathrm{n})}$ determined using the associated particle method for these reactions.

$$
k_{(\mathrm{n}, \mathrm{\alpha})}=\left(\frac{B_{\mathrm{T}(\mathrm{d}, \mathrm{n})}}{B_{\mathrm{D}(\mathrm{d}, \mathrm{n})}}\right) /\left(\frac{N_{\mathrm{T}(\mathrm{d}, \mathrm{n})}}{N_{\mathrm{D}(\mathrm{d}, \mathrm{n})}}\right)
$$

The experimental determination of these corrections is in good agreement with MonteCarlo calculations. The equipment is used to measure the neutron emission rate of radionuclide sources in the range from $10^{3} \mathrm{~s}^{-1}$ to $5 \cdot 10^{8} \mathrm{~s}^{-1}$ with the relative standard uncertainties $(k=1)$ of $0.7 \%$.

\subsection{Calorimetric method}

Radionuclide neutron sources based on the spontaneous fission of ${ }^{252} \mathrm{Cf},{ }^{244} \mathrm{Cm},{ }^{248} \mathrm{Cm}$, ${ }^{238} \mathrm{Pu},{ }^{240} \mathrm{Pu}$ etc. are used in metrology in addition to $(\alpha, \mathrm{n})$ sources. In these sources, neutrons are produced as a result of spontaneous fission events. The emission rate can be determined if the spontaneous fission activity $A_{\mathrm{f}}$, i.e. the number of fissions per unit time, of the principal radionuclide and the average number $v$ of neutrons per fission event are known with a sufficiently small uncertainty. Hence, the emission rate of such sources can in principle be 
calculated from the amount of fissionable nuclei, which can be determined by precise weighing. The most suitable nuclides for such neutron sources are ${ }^{244} \mathrm{Cm}$ and ${ }^{252} \mathrm{Cf}$, which are not fissionable by thermal neutrons, and can be purified well by modern radiochemical methods. They have a suitable half-life and a low intensity of associated $\gamma$-radiation. The neutron source strength of such sources is given by

$$
B=v \cdot\left(1+k_{1}\right) \cdot\left(1+k_{2}\right) \cdot k_{3} \cdot A_{\mathrm{f}} .
$$

Here $k_{1}$ is the number of neutrons produced by $(\alpha, n)$-reactions in the construction materials per spontaneous fission event of the principal radionuclide, $k_{2}$ is the number of neutrons resulting from fissions of other radionuclides, i.e. decay products or impurities, per fission event of the principal nuclide and $k_{3}$ denotes the correction for neutron capture by construction materials.

The fission activity $A_{\mathrm{f}}$ can be determined by measuring the thermal power $W$ released by a source using a calorimetric method. Two types of differential calorimeters were used for this measurements. One of them is a tungsten sphere $100 \mathrm{~mm}$ in diameter with a central cylindrical cavity $20 \mathrm{~mm}$ in height and diameter. The other calorimeter is a copper cylinder $25 \mathrm{~mm}$ in diameter and $55 \mathrm{~mm}$ in height. The thickness of the calorimeter walls can be varied from $2 \mathrm{~mm}$ to $8 \mathrm{~mm}$. Using this technique, the energy deposited by different kinds of radiation can be measured. Both calorimeters are placed in a multilayer thermostat. All measurements are performed in a room with a constant temperature of $(30 \pm 0.3)^{\circ} \mathrm{C}$.

This a method is very convenient because the energy release of the source results mostly from the principal radioisotope $\left({ }^{244} \mathrm{Cm}\right.$ or $\left.{ }^{252} \mathrm{Cf}\right)$. The remaining part, resulting from other radionuclide present as impurities or decay products, can be taken into account by corrections. According to the data provided by the manufacturer, the relative uncertainty of the isotopic composition at the time of packaging of the sources does not exceed $0.3 \%$. This fact minimises the uncertainty due to the influence of other isotopes. Moreover, the construction materials of the source contain only negligible amounts of light elements (except oxygen) which could increase the neutron source strength due to $(\alpha, n)$ reactions.

The total thermal power $W$ released by a source containing $n$ radionuclides is

$$
W=\sum_{i=1}^{n}\left(W_{\alpha, i}+W_{\mathrm{f}, i}\right)=\sum_{i=1}^{n}\left(A_{\alpha, i} E_{\alpha, i}+A_{\mathrm{f}, i} E_{\mathrm{f}, i}\right)
$$

where the indices ' $\mathrm{f}$ ' and ' $\alpha$ ' indicate fission events with an average energy release $E_{\mathrm{f}}$ and other decay channels with an energy release $E_{\alpha}$. With a small exception, these other channels are $\alpha$ decays. For example, the total energy release due to $\alpha$-decay and spontaneous fission is about $99.82 \%$ of the total decay energy for ${ }^{244} \mathrm{Cm}$.

The relation between $\alpha$-decay with half life $T_{\alpha}$ and spontaneous fission with half life $T_{\mathrm{f}}$ is given by

$$
A_{\mathrm{f}}=A_{\alpha} \frac{T_{\alpha}}{T_{\mathrm{f}}} .
$$


The activity $A$ of a radionuclide is related to its atomic mass $\mu$, half life $T$ and mass $m$ by

$$
A=m\left(\frac{N_{\mathrm{A}}}{\mu}\right) \frac{\ln 2}{T} .
$$

Therefore equation (7) for the total thermal power $W$ can be rearranged as

$$
W=A_{\mathrm{f}, 0}\left(\frac{T_{\mathrm{f}, 0}}{T_{\alpha, 0}} E_{\alpha, 0}+E_{\mathrm{f}, 0}+\sum_{i=1}^{n-1} \frac{A_{\alpha, i} E_{\alpha, i}+A_{\mathrm{f}, i} E_{\mathrm{f}, i}}{A_{\mathrm{f}, 0}}\right)
$$

where $A_{\mathrm{f}, 0} E_{\mathrm{f}, 0}$ is the energy release from spontaneous fission of the main radionuclide $\left({ }^{244} \mathrm{Cm}\right.$ or ${ }^{252} \mathrm{Cf}$ ) or

$$
W=A_{\mathrm{f}, 0} E_{\mathrm{f}, 0}\left(1+\frac{T_{\mathrm{f}, 0}}{T_{\alpha, 0}} \frac{E_{\alpha, i}}{E_{\mathrm{f}, i}}+\frac{T_{\mathrm{f}, 0} \mu_{0}}{E_{\mathrm{f}, 0}} m_{0} \sum_{i=1}^{n-1} \frac{m_{i}}{\mu_{i}}\left(\frac{E_{\alpha, i}}{T_{\alpha, i}}+\frac{E_{\mathrm{f}, i}}{T_{\mathrm{f}, i}}\right)\right)=A_{\mathrm{f}, 0} E_{\mathrm{f}, 0}(1+K)
$$

where $K$ is the contribution to the total energy release from all decay channels except spontaneous fission of the main radionuclide. Thus, according to the equation (6)

$$
B=\frac{W}{E_{\mathrm{f}, 0}(1+K)}\left(1+k_{1}\right)\left(1+k_{2}\right) k_{3}
$$

The coefficient $k_{2}$ for the contribution of other spontaneously fissioning isotopes to the neutron emission can be calculated from the following equation

$$
k_{2}=\frac{\mu_{0} T_{\mathrm{f}, 0}}{m_{0} v_{0}} \sum_{i=1}^{n-1} \frac{m_{i} v_{i}}{\mu_{i} T_{\mathrm{f}, i}}
$$

For calculation of the correcting factors $k_{1}, k_{2}, k_{3}, K$, the specification of the isotopic composition and the material of the source capsule by the manufacturer were used together with the nuclear data from literature $[6,7,8,9,10]$. The calorimetric method can be used to measure the neutron emission rates of radionuclide sources in the range from $10^{6} \mathrm{~s}^{-1}$ to $5 \cdot 10^{8} \mathrm{~s}^{-1}$ with a relative standard uncertainty $(k=1)$ of $0.8 \%$.

\section{Results and conclusion}

A comparison of neutron source strength measurements using the novel calorimetric method and the traditional manganese bath method is shown in Table 1. The standard uncertainty of both methods is less than $1 \%$.

The agreement of the results for the ${ }^{252} \mathrm{Cf}$ source confirms the correctness of the measurement procedure. The difference for ${ }^{244} \mathrm{Cm}$-source can be explained by an inaccuracy of the nuclear data. At present, attempts are being made to better understand this difference. 
Table 1: Neutron emission rates $B$ of different fission sources as determined with the calorimetric method or the manganese bath. The relative standard uncertainties $(k=1)$ were $0.8 \%$ for the calorimetric method and $0.55 \%$ for the manganese bath.

\begin{tabular}{|c|c|c|}
\hline \multirow{2}{*}{ source } & \multicolumn{2}{|c|}{$B / \mathrm{s}^{-1}$} \\
\cline { 2 - 3 } & calorimetric method & manganese bath \\
\hline${ }^{244} \mathrm{Cm}$ № 46.8/01 & $2.60 \cdot 10^{6}$ & $2.75 \cdot 10^{6}$ \\
\hline${ }^{244} \mathrm{Cm}$ № 46.8/02 & $2.53 \cdot 10^{6}$ & $2.67 \cdot 10^{6}$ \\
\hline${ }^{252} \mathrm{Cf}$ № 10-9/01 & $2.46 \cdot 10^{7}$ & $2.44 \cdot 10^{7}$ \\
\hline${ }^{252} \mathrm{Cf}$ № 7-9/12 & $1.12 \cdot 10^{6}$ & $1.12 \cdot 10^{6}$ \\
\hline
\end{tabular}

Internal comparisons of the facilities were carried out to confirm the correctness of the measuring procedure. During these comparisons, all the necessary corrections and constants were investigated. The neutron emission rate of four radionuclide sources with different neutron spectra were measured using the four technique discussed above. The results are given in Table 2.

Table 2: Neutron emission rates $B$ of four radionuclide sources with different spectral neutron fluences and mean neutron energies as determined with up to four different methods. The relative standard uncertainties $(k=1)$ were $0.7 \%$ for the associated particle method, $0.55 \%$ for the maganese bath, to $0.55 \%$ for gold foil activation technique and $0.8 \%$ for the calorimetric method.

\begin{tabular}{|l|c|c|c|c|}
\hline \multicolumn{1}{|c|}{ method } & $\begin{array}{c}\mathrm{Ra}-\mathrm{Be}(\gamma, \mathrm{n}) \\
\left(\begin{array}{c}\bar{E}_{\mathrm{n}} \\
100 \mathrm{keV}) \\
B / \mathrm{s}^{-1}\end{array}\right.\end{array}$ & $\begin{array}{c}{ }^{252} \mathrm{Cf} \\
\left(\begin{array}{c}\bar{E}_{\mathrm{n}} \\
2.1 \mathrm{MeV}) \\
B / \mathrm{s}^{-1}\end{array}\right.\end{array}$ & $\begin{array}{c}\mathrm{Ra}-\mathrm{Be}(\alpha, \mathrm{n}) \\
\left(\bar{E}_{\mathrm{n}} \approx 3.9 \mathrm{MeV}\right) \\
B / \mathrm{s}^{-1}\end{array}$ & $\begin{array}{c}\mathrm{Pu}-\mathrm{Be}(\alpha, \mathrm{n}) \\
\left(\bar{E}_{\mathrm{n}} \approx 4.5 \mathrm{MeV}\right) \\
B / \mathrm{s}^{-1}\end{array}$ \\
\hline associated particle & $2.87 \cdot 10^{5}$ & $1.119 \cdot 10^{6}$ & $1.083 \cdot 10^{6}$ & $1.540 \cdot 10^{7}$ \\
\hline manganese bath & $2.87 \cdot 10^{5}$ & $1.120 \cdot 10^{6}$ & $1.086 \cdot 10^{6}$ & $1.539 \cdot 10^{7}$ \\
\hline gold foil activation & & $1.118 \cdot 10^{6}$ & $1.078 \cdot 10^{6}$ & $1.533 \cdot 10^{7}$ \\
\hline calorimeter & & $1.120 \cdot 10^{6}$ & & \\
\hline
\end{tabular}

The equipment described above is a part of the Russian national standard used for the investigation of the neutron sources and fields parameters with highest accuracy. The reliability of this complex is confirmed by the fact that this equipment has been used in international comparisons since 1960.

\section{References}

[1] V.T.Shcshebolev, Publication of Metrological Institute USSR, 1962, v.69(129), p.86 (In Russian).

[2] V.T.Shcshebolev, Publication of Metrological Institute USSR, 1967, v.89(149), p.133 (In Russian).

[3] V.I.Fominykh, I.A.Yaritsyna. Publication of Metrological Institute USSR. 1967, v.89(149), p.103 (In Russian).

[4] O.L.Andreev. Atomic Energy 1964. v.16, p.3 (In Russian).

[5] E.J. Axton, Intercomparison of neutron source emission rates (1979-1984), Metrologia 27 (1990) 221-231. 
[6] V.V.Malinovsky, Semi-empirical calculation of the average number of instantaneous fission neutrons, Problems of Nuclear Science and Technology, Series: Nuclear Constants, No. 2, 1987, p.25 (In Russian).

[7] B.I.Starostov et al. Analysis and estimation of the experimental data on spectra of instantaneous fission neutrons, Problems of Nuclear Science and Technology, Series: Nuclear Constants, No. 2, 1980, p.3 (In Russian).

[8] V.V.Frolov, Nuclear physical methods for control of fissers, M.: Energoatomisdan, 1989. (In Russian)

[9] L.G.Manevich et al, Calculation of the integral characteristics of delayed neutrons, Problems of Nuclear Science and Technology. Series: Nuclear Constants. No. 2, 1988, p.3 (In Russian).

[10] Decay Data of Transactinium Nuclides, Technical Report No. 261, IAEA, Vienna, 1986. 